\title{
Limonene: Aroma of innovation in health and disease
}

\author{
A.J. Vieira, F.P. Beserra, M.C. Souza, B.M. Totti, A.L. Rozza* \\ São Paulo State University (UNESP), Institute of Biosciences, Department of Morphology, Botucatu, Brazil
}

\section{A R T I C L E I N F O}

\section{Keywords:}

Limonene

Terpenes

Essential oil

Citrus

Biological activity

\begin{abstract}
A B S T R A C T
Natural products obtained in dietary components may aid the prevention and treatment of a variety of diseases. Reports in the scientific literature have demonstrated that the consumption of terpenes is a successful alternative in the treatment of several diseases, triggering beneficial biological effects in clinical and preclinical studies. The monoterpene limonene is largely used in alimentary items, cleaning products, and it is one of the most frequent fragrances used in cosmetics formulation. The therapeutic effects of limonene have been extensively studied, proving anti-inflammatory, antioxidant, antinociceptive, anticancer, antidiabetic, antihyperalgesic, antiviral, and gastroprotective effects, among other beneficial effects in health. In this review, we collected, presented, and analyzed evidence from the scientific literature regarding the usage of limonene and its activities and underlying mechanisms involved in combating diseases. The highlighting of limonene applications could develop a useful targeting of innovative research in this field as well as the development of a limonene-based phytomedicine which could be used in a variety of conditions of health and disease.
\end{abstract}

\section{Introduction}

Plant-derived compounds show important biological properties that can be used for promotion and maintenance of health. Compounds commonly found in dietary products offer protection against diseases, and they are therapeutically important because of their properties, such as antioxidant and anti-inflammatory potential. A regular dietary intake of fruits and vegetables in the diet has protective effects against different pathologies [1].

The international definition for essential oil is the product obtained by hydrodistillation, steam distillation or dry distillation or by a suitable mechanical process without heating (for Citrus fruits) of a plant or some part of it [2]. Essential oils are volatile oily liquids, rarely colored and characterized by a strong odor [3]. This aroma results from the combination of the aromas of each molecule which composes the oil [4]. Typically, essential oils are composed by terpenes, including monoterpenes, which are the most common constituent [3].

The genus Citrus (Rutaceae) includes the most cultivated fruits in the world, such as orange, lemon, and mandarin [5]. In Citrus species, the fruit peel contains secretory cavities filled with essential oil [6]. The extraction of essential oils from Citrus fruits dates from the sixteenth century. Nowadays, the extraction process can be basically summarized in the following steps: rupture of the peel utricles by mechanical action, enabling the oil release, a water flow transports the essential oil and, at last, essential oil and water are separated by centrifugation [7].

Citrus essential oils are characterized by a volatile and a non- volatile fraction, which can be composed of more than 200 compounds. The volatile fraction is mainly composed of monoterpene and sesquiterpene hydrocarbons, also by their oxygenated derivatives, aliphatic aldehydes, alcohols and esters, forming until $99 \%$ of the essential oil. The non-volatile fraction could contain hydrocarbons, sterols, fatty acids, waxes, carotenoids, coumarins, psoralens, and flavonoids $[7,8]$.

Terpenes, the main components of essential oil, are produced by various species of plants and present diverse functions, such as defense mechanism against herbivores and pathogens and plant developmental physiology. The terpene basic chemical structure consists of an isoprene unit [9]. Terpenes can present diverse chemical structure and they are synthesized by metabolic pathways by several types of specialized plant cells $[10,11]$. Terpenes are employed in disease prevention and treatment, offering various biological effects such as antimicrobial, anti-allergenic, antioxidant, anti-inflammatory and immunomodulatory properties [12]. The terpenes large use in health can also be explained by its favorable pharmacokinetic properties, such as lipophilicity and low molecular weight $[3,13]$.

Limonene is one of the most common terpenes in nature and a major constituent of numerous essential oils from Citrus. Limonene is a colorless liquid and it exists as two optical isomers, named d- or L-limonene, and as a racemic mixture [14]. Limonene possesses a pleasant lemon-like odor, which makes it widely used as a flavor and fragrance additive in common food items, such as fruit juices, candies, chewing gums, soft drinks, ice creams. Limonene is one of the most frequent and inexpensive fragrances used in cosmetics formulation, and can be found

\footnotetext{
* Corresponding author. 250 Prof Dr Antonio Celso Wagner Zanin Street, Botucatu, SP 18618-689, Brazil.

E-mail address: arianerozza@ibb.unesp.br (A.L. Rozza).
} 
in many types of beauty products such as soaps, perfumes, shampoos, hair conditioners, and shower gels [15-17], cleaning products and ecofriendly pesticides [18]. In addition, it is considered safe for food preservation [19] and could be used as a green solvent for the extraction of natural products [20].

After oral administration, limonene is rapidly absorbed in the gastrointestinal tract, distributed and metabolized. Limonene is considered safe, presenting low toxicity to humans, without inducing mutagenic, carcinogenic, or nephrotoxic risk to humans [19].

Schmidt and Göen [21] investigated the limonene metabolism and elimination kinetics in humans. The authors found that the metabolites carveol, perillic acid, limonene-1,2-diol, and limonene-8,9-diol, but not perillyl alcohol or limonene itself, were detectable in the volunteers' blood after $5 \mathrm{~h}$ of an oral dose of limonene, evidencing a rapid first-pass metabolism. The urinary profile of metabolites is very similar to that in blood. The product of exocyclic oxidation limonene-8,9-diol was the main renal metabolite. Limonene or its metabolites also underwent respiratory elimination, leaving a characteristic odor in the exhaled air. Human limonene metabolism occurs rapidly, and the body is almost entirely cleared from the metabolites after $24 \mathrm{~h}$ of limonene ingestion.

Numerous therapeutic properties have been attributed to limonene (1-methyl-4-(1-methylethenyl) cyclohexane), a naturally occurring 10carbon cyclohexanoid monoterpene derivative [22]. The present review article covers the last ten years (2008-2017) of publications about limonene therapeutic effects published in the scientific literature, aiming to disseminate the knowledge about this useful compound and shed light in future researches regarding its biological activities in preclinical and clinical studies.

\section{Biological effects of limonene}

\subsection{Preclinical studies}

\subsubsection{Anti-inflammatory activity}

Kummer et al. [5] investigated the anti-inflammatory activity of limonene from Citrus latifolia Tanaka fruit bark essential oil using zymosan-induced peritonitis and in vitro assays. Using gas chromatography-mass spectrometry technique, the authors confirmed that limonene was the main compound present $(62 \%)$ in the essential oil of Citrus latifolia. Limonene, as well as the essential oil, did not present in vitro cytotoxicity on neutrophils derived from the peritoneal cavity of $\mathrm{BALB} / \mathrm{c}$ mice. Limonene decreased neutrophils migration when formylMet-Leu-Phe and leukotriene B4 were used as chemoattractants. Regarding in vivo assays, oral pretreatment with limonene reduced leukocytes infiltration, as well as the levels of tumor necrosis factor alpha (TNF- $\alpha$ ), a pro-inflammatory cytokine, in the peritoneal exudate after zymosan-induced peritonitis in BALB/C mice. The levels of anti-inflammatory cytokine interleukin 10 (IL-10) were not altered. The authors attributed the anti-inflammatory effect of limonene to the decreased levels of TNF- $\alpha$ and to the reduction of chemotaxis of neutrophils and leukocytes.

Macrophages play an important role in the inflammatory response, including the overproduction of pro-inflammatory cytokines and inflammatory mediators. Aiming to investigate the effects of limonene in the production of cytokines and inflammatory mediators in macrophages, Yoon et al. [23] incubated RAW 264.7 macrophages and treated them with lipopolysaccharide (LPS) and several concentrations of limonene. Limonene was able to reduce the expression of inducible nitric oxide synthase (iNOS) and cyclooxygenase (COX) as well as the production of prostaglandin $\mathrm{E} 2\left(\mathrm{PGE}_{2}\right)$, which are related to inflammatory response. The levels of pro-inflammatory cytokines TNF- $\alpha$, interleukin 1 beta (IL-1ß), and interleukin 6 (IL-6), were also decreased after the limonene treatment, in a dose-dependent manner. A cytotoxicity assay performed on HaCat keratinocytes showed no evidence of cytotoxicity. The authors suggested that limonene could be an effective anti-inflammatory agent to be used as a cosmetic or medicine in skin disorders.

Doxorubicin is a drug used for cancer treatment and has some side effects such as the production of reactive oxygen species (ROS) as well as the enhancement of the inflammatory response, causing tissue damage. Rehman et al. [24] hypothesized limonene could protect kidneys from the side effects of doxorubicin. Before doxorubicin administration, limonene was offered with the diet to Wistar rats for 20 days. Doxorubicin group presented an enhancement in TNF- $\alpha$, nuclear factor kappa $\mathrm{B}$ (NF-kB), iNOS and COX-2 expressions, inflammatory markers which were restored in limonene group. Histological analyses evidenced preservation of renal architecture and less neutrophil infiltration in the limonene-treated group. Serum blood urea nitrogen, creatinine and kidney injury molecule- 1 are nephropathy markers, which were increased in the doxorubicin group; limonene pretreatment caused a decrease in these markers levels. Limonene also reduced doxorubicin harmful action in kidneys displaying an antioxidant activity, restoring the levels of glutathione and superoxide dismutase, besides increasing the activities of catalase, glutathione peroxidase and glutathione transferase. In conclusion, doxorubicin-induced kidney damage was attenuated by a possible anti-inflammatory and antioxidant activities of limonene, indicating that this compound could be used in combination with doxorubicin.

Osteoarthritis is a degenerative disease, characterized by articular inflammation and loss of cartilage, among other complications. Rufino et al. [13] tested the activity of limonene in human chondrocytes stimulated with IL-1 $\beta$, a cell model of osteoarthritis. IL-1 $\beta$-induced nitric oxide (NO) production and the expressions of inflammatory markers, anti-catabolic and pro-anabolic genes were evaluated. Limonene, at three different concentrations, inhibited NO production and NF- $\mathrm{\kappa B}$ and p38 activation. Likewise, limonene decreased the expression of iNOS, an inflammatory marker, besides matrix metalloproteinase-1 (MMP-1) and matrix metalloproteinase-13 (MMP-13), which are catabolic genes responsible for hydrolyzing the major articular cartilage-specific matrix components. Limonene induced an increase in the expression of anticatabolic gene tissue inhibitor of metalloproteinase 1 (TIMP-1), but it was not able to increase the expression of extracellular matrix-specific genes, such as collagen II. These results granted limonene an anti-inflammatory effect on human chondrocytes by the inhibition of NO production, the decrease in iNOS expression and the non-activation of pathways linked to inflammatory response.

Investigating the effects of limonene on human eosinophilic leukemia HL-60 clone 15 cells, Hirota et al. [16] described that limonene, isolated from Citrus junos Tanaka fruit bark essential oil, decreased ROS production induced by phorbol 12-myristate 13-acetate (PMA) in dfHL-60 clone 15 cells, before and after eotaxin stimulation. Monocyte chemoattractant protein-1 (MCP-1) is a chemokine spontaneously produced by eosinophilic cells and its production increases leukocyte recruitment, triggering inflammatory or allergic reactions; limonene decreased MCP-1 production by the cells, even in the presence of a potent proteasome inhibitor. Limonene also decreased the activity of $\mathrm{NF}-\kappa \mathrm{B}$ in nuclear extracts of the cells and decreased the chemotaxis when combined with a specific p38 mitogen-activated protein kinases (MAPK) inhibitor. In summary, limonene presented an anti-inflammatory effect by decreasing ROS production, NF- $\mathrm{kB}$ activity and eosinophil migration.

We can conclude that limonene exerted an anti-inflammatory effect on in vitro and in vivo assays mainly by modulating the action of cytokines and participating in pathways that are closely linked to inflammatory response. Regarding in vitro studies, different models were performed, and the authors suggested that in vivo assays are needed to elucidate the anti-inflammatory effect of limonene.

\subsubsection{Antioxidant activity}

Oxidative stress is a consequence of the overproduction of ROS in the organism and is related to many diseases. Acting against oxidative stress, the human body has antioxidant mechanisms which have the 
capacity of attenuating damages caused by the overproduction of ROS. The ingestion of natural products with potential antioxidant activity can help the organism to counteract the oxidative stress-induced impairment.

Overproduction of ROS is one of the complications caused by diabetes mellitus in the human body. Murali et al. [25] induced diabetes using streptozotocin in Wistar male rats and treated them orally with limonene for 45 days. Antioxidant assays were performed to investigate limonene action in the diabetic condition in rat blood, kidney and liver. Diabetic rats, which received saline solution, presented high levels of thiobarbituric acid reactive substances (TBARS), lipid hydroperoxides and conjugated dienes in plasma and tissues. However, the limonene group showed a decrease in all these oxidative stress markers. Limonene group showed a significant increase of superoxide dismutase, catalase, glutathione peroxidase and glutathione transferase, which levels showed a decrease in untreated diabetic rats. The activity of glutathione and vitamin C were elevated in the limonene treated group. Limonene treatment, on histopathological analysis, led to an improvement in the liver and kidneys compared to saline treatment which presented damages in these tissues caused by diabetes. In conclusion, limonene demonstrated an antioxidant activity, attenuating oxidative stress in diabetic rats.

Similar results were observed in cultured murine lymphocytes from $\mathrm{BALB} / \mathrm{c}$ mice. Limonene at low concentrations presented a high 2,2diphenyl-1-picrylhydrazyl (DPPH) scavenging activity, an increase in the activity of catalase and peroxidase, as well as a reduction in the levels of hydrogen peroxide. It is known that superoxide dismutase converts superoxide into hydrogen peroxide and dioxygen. At high concentrations, limonene showed an enhancement of superoxide dismutase activity and in the levels of hydrogen peroxide. Cell proliferation is modulated by hydrogen peroxide and, in this study, limonene at low concentrations stimulated cell proliferation, but at high concentrations a cytostatic effect was observed. These results together suggested that limonene can protect lymphocytes from oxidative stress and at low concentrations it can stimulate cell proliferation [17].

Oxidative stress is also associated with the development of cataracts. Bai et al. [26] induced oxidative stress in human lens epithelial cells using hydrogen peroxide after limonene treatment. Hydrogen peroxide caused a decrease in cell viability, however in cells treated with limonene an increase was observed. Limonene reduced the expression of caspase-3 and caspase-9, which are proteins linked to apoptosis. The Bax/Bcl-2 ratio also plays an important role in apoptosis; limonene was able to prevent the decrease of this ratio caused by hydrogen peroxide. In order to analyze the antioxidant mechanism, which limonene plays a part, MAPK pathway was verified; limonene could inhibit the phosphorylation of p38 MAPK, which mediates oxygen peroxide-induced apoptosis. These results suggested that limonene can protect the lens epithelial cells from oxidative stress through antioxidant and anti-apoptotic pathways.

Investigating the antioxidant, cytotoxicity, genotoxic and antigenotoxic effects of limonene, Bacanli et al. [27] showed that limonene presented a decrease in cell viability in a dose-dependent way in 3-(4,5dimethylthiazol-2-yl)-2,5-diphenyltetrazolium bromide (MTT) assay. Limonene at low concentrations did not cause genotoxic damage in lymphocytes and fibroblasts in COMET assay and decreased the frequency of micronuclei in cells. In conclusion, limonene prevented lymphocytes and fibroblasts from chromosome breakage and from DNA damage, besides showing an antioxidant effect.

Ahmad and Beg [28] analyzed the hypolipidemic and antioxidant effect of limonene and thymoquinone on rats fed with atherogenic suspension. As a result, limonene presented a lipid-lowering efficacy and improved the cardiovascular disease induced by oxidative stress through a decrease in HMG-CoA reductase activity and restoration of MDA values. In addition, the intake of limonene reduced the oxidation of low density lipoprotein (LDL), small dense low density lipoprotein (sd-LDL) and large-buoyant low density lipoprotein (lb-LDL). In conclusion, limonene presented a potential atheroprotective and antioxidant effect in rats fed with atherogenic suspension.

Phenolic compounds, like limonene, showed an antioxidant activity by its free radical scavenging property. These studies above showed that limonene was able to attenuate the oxidative stress impairment on in vitro and in vivo models, confirming its antioxidant effect. Nevertheless, more studies are necessary to elucidate the pathways and mechanisms in which limonene takes part.

\subsubsection{Anticancer activity}

Citrus bergamia essential oil is $70 \%$ composed of limonene and linalyl acetate and it reduces the proliferation and the survival rate of human neuroblastoma cells SH-SY5Y [29]. Russo et al. [30] studied Citrus bergamia essential oil main compounds by individually testing them in neuroblastoma cultures, in order to identify the ones responsible for neuroblastoma cell death. The individual treatments with limonene, linalyl acetate, linalool, $\gamma$-terpinene, $\beta$-pinene and bergapten at the percentages of 0.02 and $0.03 \%$ showed a significant increase of hypodiploid events, confirming previous data of decreased cell death under these percentages of oil [29], whereas the cytotoxicity caused by the essential oil was not increased. When only limonene and linalyl acetate were assessed, results showed no accumulation of cells with sub-G1 (fragmented DNA) following a single exposure to either component. However, with combined usage, accumulation of cells with subG1 was observed, along with mitochondrial damage, cytoskeletal reorganization and decrease in cell volume. All those alterations were concentration-dependent and only occurred with combined exposure of limonene and linalyl acetate to SH-SY5Y cells. The detailed mechanism by which these monoterpenes influence cell death is yet unknown.

Investigating the antitumor effect of limonene and berberine, as well as the combination of both, Zhang et al. [31] concluded that these compounds synergistically exerted anticancer effects on human gastric carcinoma cell line MGC803. Limonene and berberine, when combined, increased apoptosis, generation of ROS and caspase-3 expression (mediator of apoptosis). Moreover, the expression of anti-apoptotic protein Bcl-2 and Rh 123 (a mitochondria-binding specific cationic fluorescent dye, related to mitochondrial transmembrane potential) presented lower levels when combined limonene and berberine than when exposed to limonene and berberine separately. The inference of the authors was that the cytotoxic effects on human gastric carcinoma cells could be obtained, in part, by the apoptosis mediated by the caspase induction and the formation of ROS.

In a similar way, Jia et al. [32] investigated the activity of limonene in LS174T human colon cancer cells. The researchers attested that the limonene, in a dose-dependent manner (variating from 0.4 to $3.2 \mu \mathrm{mol} /$ L), decreased LS174T cell viability and induced apoptosis. The effect of limonene on Akt proteins expression and activation was also analyzed; there was no expressive variation of Akt protein level, but there was a decrease in its phosphorylates residues Ser473 and Thr308, suggesting that limonene could diminish Akt activity. The treatment with limonene revealed the upregulation of pro-apoptotic Bax protein, downregulation of anti-apoptotic Bcl-2 and an increase of cytosol cytochrome $c$ level, related to the trigger of activation of procaspase-9. Furthermore, a rise in the cleavage maturation of caspase- 3 and caspase- 9 , confirmed the caspase activation, indicating that apoptosis induced by limonene is partly moderated via mitochondrial pathway. The author concluded that limonene anticancer effect was involved with the inhibition of Akt activation as well as with the mitochondrial apoptosis signaling pathway activation.

In the search for strategies to improve the efficacy of docetaxelbased treatments for hormone-refractory prostate cancer, Rabi and Bishayee [33] used limonene to enhance tumor response to docetaxel in metastatic prostate cancer, using human prostate carcinoma DU145 cells. The authors observed that Limonene and docetaxel in combination enhanced the cytotoxicity to prostate carcinoma cells, but not to normal prostate epithelial cells, which can be explained by the fact 
that normal cells express more effective antioxidant mechanisms than cancer cells. The combination of docetaxel and limonene also induced ROS generation by breaking down hydrogen peroxide $\left(\mathrm{H}_{2} \mathrm{O}_{2}\right)$-scavenging system, depletion of glutathione, and increased caspase activity, in comparison to docetaxel alone. The double therapy also induced cleavage of caspase- 9 and caspase- 3 , an apoptotic effect that was reversed after pretreatment with $\mathrm{N}$-acetylcysteine, a potent antioxidant, evidencing that antitumor effect involved ROS generation with a strong participation of caspase cascades. In this study, limonene enhanced the antitumor effect of docetaxel against prostate cancer cells without any cytotoxicity to normal prostate epithelial cells, in an effect mediated by generation of ROS and activation of caspase- 9 and caspase- 3 .

Wilson et al. [34] hypothesized if dietary chemoprevention would be effective in delaying the onset of male cancer. Thus, this research aimed to determine if limonene had a chemopreventive effect on induction and protease activity of sex accessory gland tumors in LobundWistar rats. After prostate tumor induction by nitrosomethylurea and promotion by testosterone, tumors were found in the seminal vesicles and dorsal and anterior prostate lobes from rats. However, the researchers found no difference in the distribution of tumors, site of origin or number of rats presenting metastases between control rats and limonene-treated animals. Given the importance of proteolytic activities in cancer invasion and metastasis, the activity of plasminogen activator and matrix metalloproteinases 2 and 9 were investigated. Sex accessory gland tumors and metastases expressed increased levels of plasminogen activator and metalloproteinases, but no difference between rats receiving limonene or control was noted. This study indicated that dietary supplementation with limonene could not delay or prevent the development of sex accessory gland tumors.

We can conclude that limonene presented potential anticancer activity regarding in vitro studies, but there is a need to develop a larger amount of in vivo studies to direct more conclusive answers about this effect.

\subsubsection{Antinociceptive activity}

In a recent study, Piccinelli et al. [35] orally administered limonene to mice which had previously received an intrathecal injection of glycoprotein gp120, IL-1 $\beta$ or TNF- $\alpha$, hypothesizing that limonene could prevent the hyperalgesia caused by the inflammatory cytokines or gp120. Limonene was able to decrease the production of IL-1 $\beta$ and IL10 after intrathecal injection of gp120. The activity of superoxide dismutase was increased after spinal injection of IL-1 $\beta$ or TNF- $\alpha$ in limonene treated mice, showing it can be involved in the antihyperalgesic effect since superoxide dismutase has a role in the management of pain. In conclusion, limonene was able to prevent the hyperalgesia caused by gp120, IL-1 $\beta$, and TNF- $\alpha$, probably by modulating cytokines production and superoxide dismutase expression.

Kaimoto and collaborators [36] investigated the in vivo and in vitro effect of limonene on transient receptor potential (TRP) channels, which are related to the perception of pain. Intraplantar injection of limonene in mice evoked a pain response, which was attenuated in transient receptor potential ankyrin 1 (TRPA1) null-mice, suggesting that limonene activated TRPA1, consequently triggering acute pain. However, systemic administration of limonene did not induce a pain response but induced a decrease in hydrogen peroxide-induced nociception. In DRG neurons and HEK 293 cells, when stimulated with hydrogen peroxide, limonene ceased the nociceptive effect of TRPA1 stimulation. With these results, the authors suggested that limonene has a bimodal effect on the perception of pain, mediated by TRPA1; when topically applied, limonene elicited pain, but its systemic application inhibited nociception.

With these results, it is possible to state that limonene exerted antinociceptive effect. However, more investigation is necessary to clearly understand the mechanisms related to the antinociceptive effect of limonene.

\subsubsection{Antidiabetic activity}

The increasing numbers of diabetic patients and current medicine side effects have brought the studies of potential antiglycating agents into focus. The main substances which cause complications in diabetes mellitus, such as nephropathy, arteriosclerosis, retinopathy and neuropathy, are the advanced glycation end-products (AGEs) [37]. The AGEs are responsible for alterations like physiological changes in proteins [38], intervening in its interaction with other compounds, and initiating pro-inflammatory responses [39], causing cell damage. Due to AGEs rapid formation in hyperglycemic conditions, its inhibition would prevent the mentioned complications.

Studies using Aegle marmelos leaves containing limonene showed antidiabetic activity [40]. Panaskar et al. [41] experimented on male Wistar rats with streptozotocin-induced diabetes mellitus to test the effects of limonene for antiglycative activity, utilizing chloroform extract from Aegle marmelos leaves. The usage of the extract enhanced the use of glucose (antihyperglycemic effect) and significantly decreased AGEs formation. Kidney functions were not affected, and AGEs formation was low in rats treated with the chloroform extract, concluding that the treatment could prevent complications such as nephropathy and cataract. GC-MS analysis evidenced that limonene was the main compound in chloroform extract from Aegle marmelos leaves. Purified limonene showed inhibitory effect on cataract formation, increasing reduced glutathione, catalase and superoxide dismutase activities in lens homogenate. Purified limonene also showed antiglycating activity at a much lower concentration than the currently used aminoguanidine. Authors concluded that limonene demonstrated potent antiglycative properties in very low concentrations.

Based on the results obtained by Panaskar et al. [41], Joglekar et al. [42] investigated the mechanisms of glycation inhibition by limonene. It was observed a decrease in protein unfolding in the presence of limonene. Since the limonene structure precludes the direct interaction with proteins as aminoguanidine does, it is suggested by the researchers that limonene inhibition of glycation happens by stabilizing the protein structure through hydrophobic interactions.

A more recent study by Joglekar et al. [43] assessed the combined usage of limonene and aminoguanidine. The benefits showed by the usage of aminoguanidine were many, such as the prevention of some diseases such as some nephropathy [44], atherosclerosis [45], cataract formation [46] and neuropathy [47]. The negative side to this drug, as discussed, is the large dose needed leads to toxicity, while limonene does not present this problem. When used together, however, they demonstrated a higher percentage of AGE reduction than when used separately. The presence of aminoguanidine did not prevent the binding of glucose to bovine serum albumin (BSA). The combination BSAaminoguanidine presented an affinity rate for glucose more than twenty times higher than the BSA-limonene affinity. It is clear that aminoguanidine has benefits that are outweighed by its negative effects; however, the use of limonene alongside with aminoguanidine can suppress the negative effects and provide a more effective antiglycative effect.

In brief, limonene is a potential alternative as an antiglycative agent; it can be used in lower doses and has no report of toxicity.

\subsubsection{Treatment of metabolic syndrome}

The large number of obese individuals in areas with high dietary consumption of fat has become a great concern [48], especially considering the higher risk of developing certain disorders, such as cardiovascular problems, hyperlipidemia, certain types of cancer and type 2 diabetes $[49,50]$. The nonalcoholic fatty liver disease (NAFLD), present in $75-92 \%$ of the obese population and which is the most common liver disease in the United States is also associated with obesity. Regarding those disorders brought about or aggravated by obesity, attention has been drawn to solutions involving dietary prevention and therapy, given the aggressiveness and low efficiency of medications. A greatly studied natural product that has been reported to cause positive effects 
in metabolic disorders is limonene [51].

Recent studies have reported peroxisome proliferator-activated receptors (PPARs) and liver X receptors (LXRs) as targets of citrus-derived compounds for the treatment of metabolic diseases [52-54]. Jing et al. [51] investigated limonene as one of these possible compounds, along with the assessment of limonene effects on dyslipidemia and hyperglycemia in mice, which had not yet been reported. Dyslipidemia is a disorder which presents high levels of triglyceride (TG) and total cholesterol (TC), while high-density lipoprotein (HDL) cholesterol levels are low. When limonene was applied in high-fat diet-fed mice, there was a decrease of TG and LDL cholesterol, and an increase in HDL cholesterol, while TC levels remained unaffected. The hyperglycemia was lowered in high-fat diet-fed mice in the presence of limonene. Moreover, the study also indicated a glucose tolerance built in obese mice under the use of limonene, denoting its positive effects in individuals with hyperglycemia and dyslipidemia. Regarding PPARs and LXRs, previous studies showed that the activation of PPARa with agonists can reduce TG levels and HDL cholesterol [55] and improve dyslipidemia in obese mice [56], while LXRs have a key role in signaling pathways related to hyperlipidemia [57]. Jing et al. [51] indicated that limonene activated PPAR $\alpha$, increasing its expression levels and enabling the conclusion that PPAR $\alpha$ protects against dyslipidemia. Concerning LXRs, limonene suppressed the expression of its targets in the liver, indicating that the decrease of serum lipid levels can be due to the modulating effects of LXRs signaling.

Santiago et al. [58] studied limonene effects against biochemical or histological liver alterations, in high-fat diet-fed mice and L-NAME-induced metabolic syndrome associated with NAFLD. Liver-specific enzymes such as aspartate aminotransferase (AST), alanine transaminase (ALT) and alkaline phosphatase (ALP) were observed regarding its activities in the plasma. The study results with limonene intake in high-fat diet-fed mice and high-fat diet-fed mice with L-NAME-induced metabolic syndrome showed a significant decrease in those enzymes plasma activities. NAFLD is also related to fat deposition in the liver, resulting in insulin resistance $[59,60]$. The authors observed that limonene intake lowered fat accumulation in both groups of mice. There was also a decrease in glucose blood levels and pancreatic $\beta$-cell mass with limonene supplementation. These results showed a great effect of limonene in treating metabolic syndrome associated with NAFLD.

Both studies showed limonene has a great potential regarding the treatment of metabolic syndrome and its consequences.

\subsubsection{Effects in gastrointestinal tract}

Researchers have been studying the gastroprotective and ulcer healing effects of essential oil from Citrus fruits and its major compound limonene, in the search for new compounds which could be cheap and induce no side effects.

A study carried out by Moraes et al. [61] investigated the action of limonene and essential oil from Citrus aurantium on gastric mucosa in rats, inducing ulcers through the ethanol and non-steroidal anti-inflammatory drug methods. Results demonstrated that the essential oil is composed of $97 \%$ limonene, and both compounds almost entirely avoided gastric ulcers formation at the tested doses. The gastroprotective effect could be explained by the increase of gastric mucus, which neutralizes $\mathrm{H}^{+}$concentration in the gastric juice. $\mathrm{PGE}_{2}$ plays an important role by stimulating the secretion of mucus and bicarbonate, protecting the stomach from ulcer formation. Moreover, the $\mathrm{PGE}_{2}$ levels of limonene-treated rats presented normalized values, similar to the ones in the control group. However, gastric $\mathrm{H}^{+}$secretion and glutathione levels were not altered by limonene oral treatment.

In acetic acid-induced gastric ulcers, essential oil from Citrus aurantium and limonene offered respectively $44 \%$ and $56 \%$ of healing rate in male Wistar rats. The researchers investigated the role of vascular endothelial growth factor (VEGF), proliferating cell nuclear antigen (PCNA) and cyclooxygenase 2 (COX-2), which are proteins and enzymes involved in the healing process. All the three factors were enhanced in limonene-treated rats, indicating an expressive increase of cell proliferation, increased angiogenesis and production of $\mathrm{PGE}_{2}$, ensuring the improvement of mucosal integrity [62].

Rozza and collaborators [63] analyzed the gastroprotection of essential oil from Citrus lemon and its majority compounds limonene and $\beta$-pinene. In both ethanol and indomethacin-induced gastric ulcer model, the essential oil and limonene offered effective gastroprotection; however $\beta$-pinene did not show gastroprotective activity. The authors concluded that limonene was responsible for the gastroprotective effect of Citrus lemon essential oil, stimulating the production and secretion of mucus and heat-shock protein-70. Nevertheless, limonene gastroprotective effect was neither involved with the conservation of glutathione levels, releasing of nitric oxide nor the maintenance of sulfhydryl compounds.

d'Alessio et al. [64] aimed to investigate the anti-inflammatory effect of limonene using a rat model of colitis and cell cultures of fibroblasts and enterocytes. In colitis model, limonene was administrated to Wistar rats after an injection of 2,4,6-trinitrobenzenesulfonic acid (TNBS) to induce colitis. When compared to ibuprofen, rats treated with limonene presented decreased inflammatory scores and TNF- $\alpha$ concentration in serum compared to untreated rats. Using cultured fibroblasts, limonene-containing orange peel extract was able to inhibit $\mathrm{TNF} \alpha$-induced NF- $\mathrm{\kappa B}$ translocation, suggesting that limonene acts in the $\mathrm{NF}-\kappa \mathrm{B}$ pathway. Limonene also increased epithelial resistance in colonic HT-29/B6 cell monolayers. In conclusion, limonene displayed anti-inflammatory effect in vivo, probably acting in subcellular mechanisms.

Limonene exerted protective and healing effects in the gastrointestinal tract in gastric ulcer and colitis models, serving as a propitious target to be assessed in these diseases.

\subsubsection{Effects in respiratory tract}

Acute lung injury, when associated with inflammation, is a serious disease which has high morbidity and mortality rates with no specific drug available for its treatment. Therefore, Chi and collaborators [22] hypothesized limonene could prevent acute lung injury induced by intranasal LPS in mice. As a result, limonene showed to be an effective lung protective, improving pulmonary function through its anti-inflammatory activity. Limonene was able to decrease inflammatory neutrophil infiltration and the subsequent activity of myeloperoxidase (MPO). Inflammatory cytokines such as TNF- $\alpha$, IL-1 $\beta$ and IL-6 are considered therapeutic targets in acute lung injury; limonene treatment decreased the activity of these cytokines. Some inflammatory mechanisms were investigated and limonene was able to attenuate the activation of NF- $\mathrm{kB}$, ERK, JNK, and p38 MAPK signaling pathways, showing an anti-inflammatory effect on the prevention of LPS-induced acute lung injury and evidencing a potential new bioactivity for limonene.

Bronchial asthma is a chronic inflammatory disease which affects 300 million people worldwide. This illness is clinically characterized by airway obstruction in response to allergens, chronic eosinophilic airway inflammation, mucus hypersecretion, and airway remodeling and nonspecific airway hyperresponsiveness [65]. Individuals susceptible to asthma or allergic airway disease are considered more sensitive to inhaled irritant agents due to these airway abnormalities. Therefore, a study performed by Hirota et al. [66] aimed to evaluate whether limonene could reduce allergic airway and improve the asthma symptoms in Dermatophagoides farinae-induced (DER) airway inflammation model. The results obtained showed that oral treatment with limonene significantly decreased the production of immunoglobulin E (IgE) (important marker of allergic inflammation), representative Th- 2 cell type (responsible for producing pro-inflammatory cytokines), chemoattractant cytokines MCP-1 (important for the influx of inflammatory cells) and TGF- $\beta 1$ (associated with the development of airway remodeling in asthma). Histopathological changes also showed that treatment with limonene efficiently reduced the influx of eosinophils into the lungs, airway fibrosis and mitigated the collagen 
deposition. In addition, limonene treatment was able to attenuate the bronchoconstriction caused by the administration of acetylcholine in DER mice, showing involvement with cholinergic receptors. These results showed that limonene possesses a potent therapeutic effect on the allergic airway and for asthma. However, further researches are necessary to ensure these same beneficial effects in asthmatic human patients.

Several epidemiological studies have described associations between bronchial asthma attacks and high levels of air pollution, such as ozone $\left(\mathrm{O}_{3}\right)$. Thus, Hansen et al. [67] verified whether the inhalation of the reaction products of $\mathrm{O}_{3}$ and limonene, as well as limonene and lowlevel $\mathrm{O}_{3}$, were able to provoke airway inflammation and allergic sensitization in a subchronic mouse inhalation model and allergen ovalbumin (OVA) in BALB/cJ mice. The results showed that the mixture of $\mathrm{O}_{3}$ and limonene stimulated the OVA-specific IgE production compared to limonene and $\mathrm{O}_{3}$ separately, suggesting that this reactive mixture provokes an adjuvant effect on allergic sensitization. Although limonene has decreased IL-5 levels and surfactant protein D (SP-D) expression, this monoterpene did not decrease neutrophils and eosinophils infiltration. On the other hand, its mixture decreased the number of inflammatory cells, neutrophils, and eosinophils, compared to the control group, which showed that this mixture did not cause any allergic lung inflammation. This study does not support the fact that the mixture of $\mathrm{O}_{3}$ and limonene in indoor air promotes allergic airway inflammation. The authors reinforce that the inflammation is a characteristic of allergic asthma and its absence in the present study possibly occurred due to the antioxidant effects of limonene. Another study performed by the same group of researchers [68] investigated whether limonene, $\mathrm{O}_{3}$ or the $\mathrm{O}_{3}$-limonene reaction mixture could aggravate allergic lung inflammation in BALB/cJ mice. This study showed that mice exposed via inhalation for three consecutive days to clean air, ozone, limonene or an $\mathrm{O}_{3}$-limonene reaction mixture, did not suffer exacerbation in the airway allergy by any of these exposures, and mice which inhaled limonene or the reaction mixture presented a decreased allergic inflammation. Although limonene itself did not show any reduction in cell infiltration, the reaction mixture decreased the infiltration of inflammatory cells, pro-inflammatory cytokines and SP-D expression. This research concludes that the reduction of allergic airway responses after short-term exposure to limonene and mixture reaction possibly might be attributed to the antioxidant and anti-inflammatory properties of this monoterpene.

\subsubsection{Other activities}

Peripheral nerve injury can lead to harmful consequences, including depression and associated cognitive and emotional comorbidities [69]. A study performed by Piccinelli et al. [70] showed antidepressant and antihyperalgesic effects of limonene on spared nerve injury (SNI) model of neuropathic pain in rats. In this study, rats were treated for up to 15 days with limonene and the results demonstrated antihyperalgesic activity in the SNI-induced mechanical hyperalgesia as well as decreased depressive-like behavior, however it was not able to prevent the sensitivity to a cold stimulus in 10 and 15 days after SNI and not even change locomotion activity evaluated in open-field test in rats. The authors consider this study as a promising field for the discovery of new anti-hyperalgesic and/or antidepressant agents. Limonene also showed a potential anti-stress effect in a study performed by d'Alessio et al. [71]. The aim of this study was to evaluate the effect of oral treatment with limonene and its metabolite perillyl alcohol in a rat model submitted to non-pathologic mild stress conditions. The findings are complementary to a previous report [72] which, in turn, showed a potential effect of limonene and perillyl alcohol on the emotional aspects of stress analyzed through a functional observation battery, including behavioral, neurological and physiological parameters. This additional study also showed that the oral administration of limonene and its metabolite perillyl alcohol showed a significant anti-stress effect, observed for a series of behavioral and physiological changes under the influence of the nervous system (ortho-parasympathetic system), even though no alteration of neurological parameters had been verified. Although these tested substances showed almost the same results, the effects were more pronounced and significant in rats fed with limonene than with perillyl alcohol, suggesting the importance of the endogenous metabolism in the effect of this monoterpene.

The efficiency of limonene and its metabolites limonene-1-2-diol and perillic acid as immunomodulatory agents was tested on the immune system function using an isolated in vitro assay system [73]. This study revealed that limonene and its metabolites strongly reduced the production of interferon gamma (IFN- $\gamma$ ), interleukin 2 (IL-2), TNF- $\alpha$, interleukin 4 (IL-4) and interleukin 13 (IL-13) by $\mathrm{CD}^{+}{ }^{+} \mathrm{CD} 4^{+}$cells, and the production of IFN- $\gamma$, IL-2, and TNF- $\alpha$ by $\mathrm{CD} 3{ }^{+} \mathrm{CD} 8{ }^{+} \mathrm{T}$ cells. Limonene treatment inhibited the production of TH1 and TH2 cytokines by activated $\mathrm{T}$ cells. Limonene and its metabolites also showed cytotoxic potential in isolated $\mathrm{CD} 4^{+}$and $\mathrm{CD}^{+} \mathrm{T}$ lymphocytes when treated with higher doses, while in lower concentrations it showed no effect on $\mathrm{T}$ cell viability. These immunosuppressive effects, as well as the safe use and clinical efficacy, must be considered when utilizing the molecule for the therapeutic and commercial applications.

Another study carried out by d'Alessio et al. [74] investigated the anti-inflammatory, wound-healing and anti-angiogenic properties of limonene and its major metabolite $\mathrm{POH}$, in two in vivo models of 12-Otetradecanoylphorbol-13-acetate (TPA)-induced dermatitis and mechanical skin injury. The results showed that both limonene and its metabolite decreased the severity and extension of TPA-induced skin injuries compared to negative control. In this same TPA model, limonene and $\mathrm{POH}$-treated animals showed decreased serum concentrations of pro-inflammatory cytokines IL- 6 and TNF- $\alpha$, confirming the potent anti-inflammatory effect in this skin inflammatory process. In the cutaneous wound-healing model, $\mathrm{POH}$ showed to be more efficient than limonene by improving the tissue regeneration and neovascularization which strongly contribute to the tissue repairing process. Therefore, limonene and $\mathrm{POH}$ confirmed the anti-inflammatory and wound healing effects in animal models, especially for decreasing both inflammation and neoangiogenesis, suggesting a direct action of these monoterpenes on epithelial cells.

It is known that natural products are still major sources of innovative therapeutic agents for various conditions, including viral diseases. In this regard, the antiviral activity of limonene and $\beta$-pinene against the herpes simplex type 1 (HSV-1) was evaluated, elucidating its mechanisms of antiviral action. The results indicated high antiherpetic activity of both monoterpenes which were able to completely suppress the viral replication. The mechanism of antiviral action showed minor effects on the viral infection when host cells were pretreated with drugs prior to the infection. Conversely, when both monoterpenes were added to the HSV-1 infected cells after the penetration of the viruses into cells, plaque formation was not decreased showing direct interaction with free virus particles. These data suggest that limonene and $\beta$-pinene are promising substances for application in recurrent herpes labialis, making it possible to carry out more researches applied to the area [75].

Another major challenge to present-day medicine, mainly due to the lack of sufficient evidence, is to study the efficacy and safety of medications during pregnancy. As already mentioned, although limonene possesses chemotherapeutic, antinociceptive, anti-inflammatory and antioxidant effects, it modifies the myometrial relaxing effect of terbutaline and decreases its cervical resistance-enhancing effect [76]. Since there were no reports in the literature on the effect of limonene on myometrial smooth muscle, Hajagos-Tóth et al. [77] studied the effects of $\mathrm{d}$ - and L-limonene on the pregnant rat myometrium in vitro and how these effects were modified by other drugs, such as nifedipine, an L-type $\mathrm{Ca}^{2+}$ channel blocker; paxilline, a selective $\mathrm{Ca}^{2+}$-activated potassium (BKCa) channel blocker; tetraethyl-ammonium (TEA), a non-selective $\mathrm{K}^{+}$channel blocker, and theophylline, a non-selective adenosine receptor antagonist. The study showed an antioxidant effect of limonene by decreasing the inhibitory effect of methylglyoxal (MGO), an 


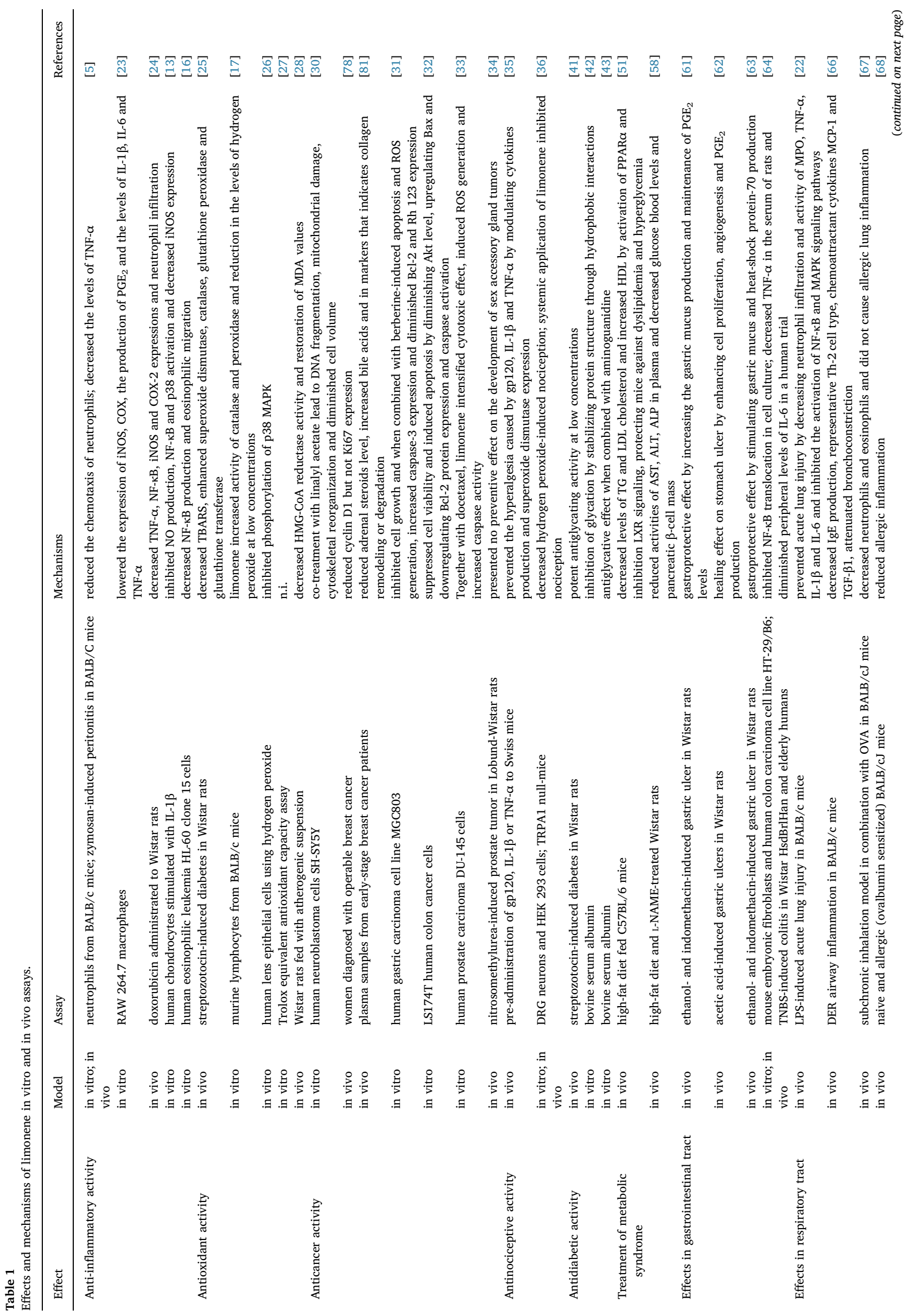


indicator of oxidative damage and both d- and L-limonene caused myometrial contraction in a dose-dependent way. The pre-treatment with nifedipine and TEA decreased the myometrial effect of limonene, while the myometrial contraction in the presence of paxilline was not observed. These findings suggest that the mechanism of limonene can be involved in increasing intracellular calcium and BKCa channels. According to the findings of this study, it is likely that limonene induces the uterus contractility and its use should be avoided during pregnancy. Although the authors did not perform the contractility studies on human myometrium, they hypothesized that the same effect can be reproduced in humans.

\subsection{Clinical studies}

Limonene-containing orange peel extract (OPE) was used as a diet supplement by healthy elderly humans (65-85 years old) from three countries. In this study, it was compared people who had received daily a soft gel capsule containing OPE for 56 days with people without any treatment. As results, there were found no differences between day one and day 56 of treatment, but the authors established an inflammatory score, in which levels of IL- 6 were significantly decreased after 56 days of treatment. The authors concluded that limonene could be used as a diet supplementation in anti-inflammatory therapies [64].

Miller et al. [78] studied the effects of limonene in breast cancer in recently diagnosed women, hypothesizing an extensive distribution of the compound in breast tissues that could have preventive effects. By studying 40 women with $2 \mathrm{~g}$ of limonene ingested daily for 2-6 weeks, the average limonene concentration found in breast tissues was $41.3 \mu \mathrm{g} / \mathrm{g}$. The authors observed a decrease in the expression of cyclin D1, a protein involved in cell cycle progression. Its overexpression would result in rapid proliferation [79,80]. However, its decrease was not accompanied by a decrease in the expression of proliferation marker Ki67. The researchers hypothesized that the recruitment of patients in a more advanced state of the disease or with a longer intervention would provide a higher percentage of the marker and more concrete results involving limonene activity in breast cancer would be observed.

To deepen the knowledge of limonene effects in mammary cancer, a more recent study by Miller et al. [81] analyzed plasma metabolites using samples collected in the previous study. The authors observed a decrease in adrenal steroid levels, which were proved to induce proliferation effects in high concentrations [82]. An increase in bile acids was also observed, which acts as a signaling molecule through farnesoid $\mathrm{X}$ receptor (FXR). The FXRs were assessed in human breast cancer cell lines [83], therefore indicating that possible alterations in bile could have some effect on the tissue. An increase in the markers which indicate collagen remodeling or degradation, such as glycine, proline, hydroxyproline and proline-hydroxyproline, was also observed. These authors concluded that limonene anti-cancer properties are consistent with previous studies and should be further assessed.

Clinical trials using limonene are yet incipient and do not allow many conclusions about its effects specifically in human health and diseases. But we consider that this can be an advantageous point since there is an open door for new clinical trials which could be developed in the search for new drugs and treatments for common diseases.

We have highlighted the preclinical and clinical data, in order to examine limonene's efficacy in cancer, diabetes, respiratory and gastrointestinal disorders, metabolic syndrome, among others. A summary of the findings for each disorder is presented in Table 1, as well as other information described in the text such as models, assay and their mechanisms of action.

\section{Conclusion and perspectives}

Nowadays, researchers have made extensive efforts on natural products as an alternative or complementary medicine because of 
attractive factors such as relative easy acquisition, efficacy, safety, and low cost. The results reported here validate the growing interest in the biological activities of limonene. We reported and analyzed more than sixty articles published in the last ten years regarding different effects offered by limonene. It can be affirmed that limonene presents antiinflammatory, antidiabetic, antioxidant, anticancer, antiallergic, antinociceptive, and anti-stress activities, besides positive effects in the treatment of gastric ulcer, colitis, asthma and airway inflammation, among other activities. The high availability of limonene in nature, its safety profile and its wide mechanism of action make this monoterpene a promising alternative to conventional therapeutic drugs. We expect that the considerable amount of evidence discussed here contributes to increase the knowledge of biological effects and mechanisms of action exerted from limonene and could direct future preclinical and clinical studies using limonene alone or in combination with other drugs, as an alternative or complementary phytomedicine.

\section{Conflicts of interest}

None.

\section{Transparency document}

Transparency document related to this article can be found online at http://dx.doi.org/10.1016/j.cbi.2018.02.007.

\section{References}

[1] K. Singletary, Diet, natural products and cancer chemoprevention, J. Nutr. 130 (2) (2000) 465S-466S.

[2] P. Rubiolo, B. Sgorbini, E. Liberto, C. Cordero, C. Bicchi, Essential oils and volatiles: sample preparation and analysis, Flavour Fragrance J. 25 (2010) 282-290.

[3] M.G. Miguel, Antioxidant and anti-inflammatory activities of essential oils: a short review, Molecules 15 (2010) 9252-9287.

[4] N.S. Sangwan, A.H.A. Farooqui, F. Shabih, R.S. Sangwan, Regulation of essential oil production in plants, Plant Growth Regul. 34 (2001) 3-21.

[5] R. Kummer, F.C. Fachini-Queiroz, C.F. Estevão-Silva, R. Grespan, E.L. Silva, C.A. Bersani-Amado, R.K.N. Cuman, Evaluation of anti-inflammatory activity of citrus latifolia Tanaka essential oil and limonene in experimental mouse models, Evid. base Compl. Alternative Med. 2013 (2013) 8. Article ID 859083.

[6] S.S. Voo, H.D. Grimes, B.M. Lange, Assessing the biosynthetic capabilities of secretory glands in Citrus peel, Plant Physiol. 159 (2012) 81-94.

[7] G. Dugo, A. Di Giacomo, Citrus: the Genus Citrus, Taylor and Francis, London, 2002.

[8] P.Q. Tranchida, I. Bonaccorsi, P. Dugo, L. Mondello, G. Dugo, Analysis of Citrus essential oils: state of the art and future perspectives. A review, Flavour Fragrance J. 27 (2012) 98-123.

[9] R. Mewalal, D.K. Rai, D. Kainer, F. Chen, C. Külheim, G.F. Peter, G.A. Tuskan, Plantderived terpenes: a feedstock for specialty biofuels, Trends Biotechnol. 35 (2017) 227-240.

[10] K.G. Zulak, J. Bohlmann, Terpenoid biosynthesis and specialized vascular cells of conifer defense, J. Integr. Plant Biol. 52 (2010) 86-97.

[11] K.S. Cho, Y.R. Lim, K. Lee, J. Lee, J.H. Lee, I.S. Lee, Terpenes from forests and human health, Toxicol. Res. 33 (2017) 97-106.

[12] N. Theis, M. Lerdau, The evolution of function in plant secondary metabolites, Int. J. Plant Sci. 164 (S3) (2003) S93-S102.

[13] A.T. Rufino, M. Ribeiro, C. Sousa, F. Judas, L. Salgueiro, C. Cavaleiro, A.F. Mendes, Evaluation of the anti-inflammatory, anti-catabolic and pro-anabolic effects of Ecaryophyllene, myrcene and limonene in a cell model of osteoarthritis, Eur. J. Pharmacol. 750 (2015) 141-150.

[14] R.S. Zulaikha, S.I.S. Norkhadijah, S.M. Praveena, Hazardous ingredients in cosmetics and personal care products and health concern: a review, Publ. Health Res. 5 (2015) 7-15.

[15] A.F. Filipsson, J. Bard, S. Karlsson, Limonene, fifth ed., World Health Organization, 1998, p. 32.

[16] R. Hirota, N.N. Roger, H. Nakamura, H.S. Song, M. Sawamura, N. Suganuma, Antiinflammatory effects of limonene from yuzu (citrus junos Tanaka) essential oil on eosinophils, J. Food Sci. 75 (2010) H87-H92.

[17] D. Roberto, P. Micucci, T. Sebastian, F. Graciela, C. Anesini, Antioxidant activity of limonene on normal murine lymphocytes: relation to $\mathrm{H} 2 \mathrm{O} 2$ modulation and cell proliferation, Basic Clin. Pharmacol. Toxicol. 106 (2010) 38-44.

[18] W.W. Nazaroff, C.J. Weschler, Cleaning products and air fresheners: exposure to primary and secondary air pollutants, Atmos. Environ. 38 (2004) 2841-2865.

[19] J. Sun, D-Limonene: safety and clinical applications, Alternative Med. Rev. 12 (3) (2007) 259-264.

[20] S. Chemat, V. Tomao, F. Chemat, Limonene as Green solvent for extraction of natural products, in: A. Mohammad, Inamuddin (Eds.), Green Solvents I: Properties and Applications in Chemistry, Springer Netherlands, Amsterdam, 2012, pp. 175-186.

[21] L. Schmidt, T. Göen, R-Limonene metabolism in humans and metabolite kinetics after oral administration, Arch. Toxicol. 91 (2017) 1175-1185.

[22] G. Chi, M. Wei, X. Xie, L.W. Soromou, F. Liu, S. Zhao, Suppression of MAPK and NF$\mathrm{kB}$ pathways by limonene contributes to attenuation of lipopolysaccharide-induced inflammatory responses in acute lung injury, Inflammation 36 (2) (2013) 501-511.

[23] W.J. Yoon, N.H. Lee, C.G. Hyun, Limonene suppresses lipopolysaccharide-induced production of nitric oxide, prostaglandin E2, and pro-inflammatory cytokines in RAW 264.7 macrophages, J. Oleo Sci. 59 (8) (2010) 415-421.

[24] M.U. Rehman, M. Tahir, A.Q. Khan, R. Khan, Oday-O-Hamiza, A. Lateef, S.K. Hassan, S. Rashid, N. Ali, M. Zeeshan, S. Sultana, D-limonene suppresses doxorubicin-induced oxidative stress and inflammation via repression of COX-2, iNOS, and NFkB in kidneys of Wistar rats, Exp. Biol. Med. 239 (4) (2014) 465-476.

[25] R. Murali, A. Karthikeyan, R. Saravanan, Protective effects of d-limonene on lipid peroxidation and antioxidant enzymes in streptozotocin-induced diabetic rats, Basic \& Clin. Pharmacol. Toxicol. 112 (2013) 175-181 2013.

[26] J. Bai, Y. Zheng, G. Wang, P. Liu, Protective effect of d-limonene against oxidative stress-induced cell damage in human lens epithelial cells via the p38 pathway, Oxid. Med. Cell. Long. 2016 (2016) 12. Article ID 5962832.

[27] M. Bacanl1, A.A. Başaran, N. Başaran, The antioxidant and antigenotoxic properties of citrus phenolics limonene and naringin, Food and Chem. Toxicol. 81 (2015) $160-170$

[28] S. Ahmad, Z.H. Beg, Hypolipidemic and antioxidant activities of thymoquinone and limonene in atherogenic suspension fed rats, Food Chem. 138 (2013) 1116-1124.

[29] L. Berliocchi, A. Ciociaro, R. Russo, M.G. Cassiano, F. Blandini, D. Rotiroti, L.A. Morrone, M.T. Corasaniti, Toxic profile of bergamot essential oil on survival and proliferation of SH-SY5Y neuroblastoma cells, Food and Chem. Toxicol. 49 (11) (2011) 2780-2792.

[30] R. Russo, A. Ciociaro, L. Berliocchi, M.G. Cassiano, L. Rombolà, S. Ragusa, G. Bagetta, F. Blandini, M.T. Corasaniti, Implication of limonene and linalyl acetate in cytotoxicity induced by bergamot essential oil in human neuroblastoma cells, Fitoterapia 89 (2013) 48-57.

[31] X.Z. Zhang, L. Wang, D.W. Liu, G.Y. Tang, H.Y. Zhang, Synergistic inhibitory effect of berberine and d-limonene on human gastric carcinoma cell line MGC803, J. Med. Food 17 (9) (2014) 955-962.

[32] S.S. Jia, G.P. Xi, M. Zhang, Y.B. Chen, B. Lei, X.S. Dong, Y.M. Yang, Induction of apoptosis by D-limonene is mediated by inactivation of Akt in LS174T human colon cancer cells, Oncol. Rep. 29 (1) (2013) 349-354.

[33] T. Rabi, A. Bishayee, d-Limonene sensitizes docetaxel-induced cytotoxicity in human prostate cancer cells: generation of reactive oxygen species and induction of apoptosis, J. Carcinogen. 8 (9) (2009).

[34] M.J. Wilson, B.R. Lindgren, A.A. Sinha, The effect of dietary supplementation with limonene or myo-inositol on the induction of neoplasia and matrix metalloproteinase and plasminogen activator activities in accessory sex organs of male Lobund-Wistar rats, Exp. Mol. Pathol. 85 (2008) 83-89.

[35] A.C. Piccinelli, P.N. Morato, M.S. Barbosa, J. Croda, J. Sampson, X. Kong, E.C. Konkiewitz, E.B. Ziff, J. Amaya-Farfan, C.A.L. Kassuya, Limonene reduces hyperalgesia induced by gp120 and cytokines by modulation of IL-1 $\beta$ and protein expression in spinal cord of mice, Life Sci. 174 (2017) 28-34.

[36] T. Kaimoto, Y. Hatakeyama, K. Takahashi, T. Imagawa, M. Tominaga, T. Ohta, Involvement of transient receptor potential A1 channel in algesic and analgesic actions of the organic compound limonene, Euro. J. Pain 20 (7) (2016) 1155-1165.

[37] R. Singh, A. Barden, T. Mori, L. Beilin, Advanced glycation end-products: a review, Diabetologia 44 (2) (2001) 129-146.

[38] R.G. Paul, A.J. Bailey, The effect of advanced glycation end-product formation upon cell-matrix interactions, Int. J. Biochem. Cell Biol. 31 (6) (1999) 653-660.

[39] M.A. Schmidt, D.M. Stern, Receptor for age (RAGE) is a gene within the majo histocompatibility class III region: implications for host response mechanisms in homeostasis and chronic disease, Front.Biosci. 6 (2001) d1151-1160.

[40] N. Kamalakkannan, P. Prince, Antidiabetic and anti-oxidant activity of Aegle marmelos extract in streptozotocin-induced diabetic rats, Pharma. Biol. 42 (2004) $125-130$.

[41] S.N. Panaskar, M.M. Joglekar, S.S. Taklikar, V.S. Haldavnekar, A.U. Arvindekar, Aegle marmelos Correa leaf extract prevents secondary complications in streptozotocin-induced diabetic rats and demonstration of limonene as a potent antiglycating agent, J. Pharm. Pharmacol. 65 (2013) 884-894.

[42] M.M. Joglekar, S.N. Panaskar, A.D. Chougale, M.J. Kulkarni, A.U. Arvindekar, A novel mechanism for antiglycative action of limonene through stabilization of protein conformation, Mol. BioSys. 9 (2013) 2463-2472.

[43] M.M. Joglekar, L.N. Bavkar, S. Sistla, A.U. Arvindekar, Effective inhibition of protein glycation by combinatorial usage of limonene and aminoguanidine through differential and synergistic mechanisms, Int. J. Biol. Macromol. 99 (2017) 563-569.

[44] D. Edelstein, M. Brownlee, Mechanistic studies of advanced glycosylation end product inhibition by aminoguanidine, Diabetes 41 (1992) 26-29.

[45] T.S. Liparota, M. Cooper, D. Papazoglou, B. Clarke, G. Jerums, Retardation by aminoguanidine of development of albuminuria, mesangial expansion, and tissue fluorescence in streptozocin-induced diabetic rat, Diabetes 40 (1991) 1328-1334.

[46] Y.M. Li, M. Steffes, T. Donnelly, C. Liu, H. Fuh, J. Basgen, R. Bucala, H. Vlassara, Prevention of cardiovascular and renal pathology of aging by the advanced glycation inhibitor aminoguanidine, Proc. Natl. Acad. Sci. U.S.A. 93 (1996) 3902-3907.

[47] T. Nabekura, Y. Koizumi, M. Nakao, M. Tomohiro, M. Inomata, Y. Ito, Delay of cataract development in hereditary cataract UPL rats by disulfiram and aminoguanidine, Exp. Eye Res. 76 (2003) 169-174.

[48] B. Canbakan, V. Tahan, H. Balci, I. Hatemi, B. Erer, G. Ozbay, N. Sut, M. Hacibekiroglu, N. Imeryuz, H. Senturk, Leptin in nonalcoholic fatty liver disease, 
Ann. Hepatol. 7 (2008) 249-254.

[49] Y.C. Wang, K. McPherson, T. Marsh, S.L. Gortmaker, M. Brown, Health and economic burden of the projected obesity trends in the USA and the UK, Lancet 378 (2011) 815-825.

[50] J.W. Yun, Possible anti-obesity therapeutics from nature - a review, Phytochemistry 71 (2010) 1625-1641.

[51] L. Jing, Y. Zhang, S. Fan, M. Gu, Y. Guan, X. Lu, C. Huang, Z. Zhou, Preventive and ameliorating effects of citrus d-limonene on dyslipidemia and hyperglycemia in mice with high-fat diet-induced obesity, Euro. J. Pharmacol. 715 (2013) 46-55.

[52] J. Goldwasser, P.Y. Cohen, E. Yang, et al., Transcriptional regulation of human and rat hepatic lipid metabolism by the grapefruit flavonoid naringenin: role of PPARa, PPAR $y$ and LXRa, PLoS One 5 (8) (2010) 1-9.

[53] G.S. Kim, H.J. Park, J.H. Woo, M.K. Kim, P.O. Koh, W. Min, Y.G. Ko, C.H. Kim, C.K. Won, J.H. Cho, Citrus aurantium flavonoids inhibit adipogenesis through the Akt signaling pathway in 3T3-L1 cells, BMC Comp. Alt. Med. 12 (2012) 31.

[54] A.K. Sharma, S. Bharti, S. Ojha, J. Bhatia, N. Kumar, R. Ray, S. Kumari, D.S. Arya Up-regulation of PPAR $\gamma$, heat shock protein-27 and -72 by naringin attenuates insulin resistance, $\beta$-cell dysfunction, hepatic steatosis and kidney damage in a rat model of type 2 diabetes, Br. J. Nutr. 106 (11) (2011) 1713-1723.

[55] M. Yoon, The role of PPARa in lipid metabolism and obesity: focusing on the effects of estrogen on PPARa actions, Pharmacol. Res. 60 (2009) 151-159.

[56] Y.-I. Kim, S. Hirai, T. Goto, C. Ohyane, H. Takahashi, T. Tsugane, C. Konishi, T. Fujii, S. Inai, Y. Iijima, K. Aoki, D. Shibata, N. Takahashi, T. Kawada, Potent PPAR $\alpha$ activator derived from tomato juice, 13-oxo-9,11-octadecadienoicacid, decreases plasma and hepatic triglyceride in obese diabetic mice, PLoS One 7 (2) (2012).

[57] P. Tontonoz, D.J. Mangelsdorf, Liver X receptor signaling pathways in cardiovascular disease, Mol. Endocrinol. 17 (2003) 985-993.

[58] J.V.A. Santiago, J. Jayachitra, M. Shenbagam, N. Nalini, Dietary d-limonene alleviates insulin resistance and oxidative stress-induced liver injury in high-fat diet and L-NAME-treated rats, Euro. J. Nutr. 51 (2012) 57-68.

[59] N.M. Delzenne, N.A. Hernaux, H.S. Taper, A new model of acute liver steatosis induced in rats by fasting followed by refeeding a high carbohydrate-fat free diet, J. Hepatol. 26 (1997) 880-885.

[60] S. Parekh, F.A. Anania, Abnormal lipid and glucose metabolism in obesity: implications for nonalcoholic fatty liver disease, Gastroenterology 132 (6) (2007) 2191-2207.

[61] T.M. Moraes, H. Kushima, F.C. Moleiro, R.C. Santos, L.R.M. Rocha, M.O. Marques, W. Vilegas, C.A. Hiruma-Lima, Effects of limonene and essential oil from Citrus aurantium on gastric mucosa: role of prostaglandins and gastric mucus secretion, Chemico-Biol. Inter. 180 (3) (2009) 499-505.

[62] T.M. Moraes, A.L. Rozza, H. Kushima, C.H. Pellizzon, L.R.M. Rocha, C.A. HirumaLima, Healing actions of essential oils from Citrus aurantium and d-limonene in the gastric mucosa: the roles of VEGF, PCNA, and COX-2 in cell proliferation, J. Med. Food 16 (12) (2013) 1162-1167.

[63] A.L. Rozza, T.M. Moraes, H. Kushima, A. Tanimoto, M.O.M. Marques, T.M. Bauab, C.A. Hiruma-Lima, C.H. Pellizzon, Gastroprotective mechanisms of Citrus lemon (Rutaceae) essential oil and its majority compounds limonene and $\beta$-pinene: involvement of heat-shock protein-70, vasoactive intestinal peptide, glutathione, sulfhydryl compounds, nitric oxide and prostaglandin E2, Chemico-Biol. Inter. 189 (1) (2011) 82-89.

[64] P.A. d'Alessio, R. Ostan, J.F. Bisson, J.D. Schulzke, M.V. Ursini, M.C. Béné, Oral administration of d-limonene controls inflammation in rat colitis and displays antiinflammatory properties as diet supplementation in humans, Life Sci. 92 (24) (2013) 1151-1156.

[65] B.N. Lambrecht, H. Hammad, The immunology of asthma, Nature Immunol. 16
(2015) 45-56.

[66] R. Hirota, H. Nakamura, S.A. Bhatti, N.R. Ngatu, B.A. Muzembo, N. Dumavibhat, M. Eitoku, M. Sawamura, N. Suganuma, Limonene inhalation reduces allergic airway inflammation in Dermatophagoides farinae-treated mice, Inhal. Toxicol. 24 (6) (2012) 373-381.

[67] J.S. Hansen, G.D. Nielsen, J.B. Sørli, P.A. Clausen, P. Wolkoff, S.T. Larsen, Adjuvant and inflammatory effects in mice after subchronic inhalation of allergen and ozoneinitiated limonene reaction products, J. Toxicol. Environ. Health, Part A 76 (19) (2013) 1085-1095.

[68] J.S. Hansen, A.W. Nørgaard, I.K. Koponen, J.B. Sørli, M.D. Paidi, S.W.K. Hansen, P.A. Clausen, G.D. Nielsen, P. Wolkoff, S.T. Larsen, Limonene and its ozone-initiated reaction products attenuate allergic lung inflammation in mice, J. Immunotoxicol. 13 (6) (2016) 793-803.

[69] S. Alvarado, M. Tajerian, M. Millecamps, M. Suderman, L.S. Stone, M. Szyf, Peripheral nerve injury is accompanied by chronic transcriptome-wide changes in the mouse prefrontal cortex, Mol. Pain 9 (2013) 21.

[70] A.C. Piccinelli, J.A. Santos, E.C. Konkiewitz, S.A. Oesterreich, A.S.N. Formagio, J. Croda, E.B. Ziff, C.A.L. Kassuya, Antihyperalgesic and antidepressive actions of (R)-(+)-limonene, $\alpha$-phellandrene, and essential oil from Schinus terebinthifolius fruits in a neuropathic pain model, Nutr. Neurosci. 14 (5) (2015) 217-224.

[71] P.A. d'Alessio, J.F. Bisson, M.C. Béné, Anti-stress effects of d-Limonene and its metabolite perillyl alcohol, Rejuvenation Res. 17 (2) (2014) 145-149.

[72] J.F. Bisson, C. Menut, P.A. d'Alessio, Anti-inflammatory senescence actives 5203-L molecule to promote healthy aging and prolongation of lifespan, Rejuvenation Res. 11 (2) (2008) 399-407.

[73] C.M. Lappas, N.T. Lappas, D-Limonene modulates T lymphocyte activity and viability, Cell. Immunol. 279 (1) (2012) 30-41.

[74] P.A. d'Alessio, M. Mirshahi, J.F. Bisson, M.C. Béné, Skin repair properties of d-limonene and perillyl alcohol in murine models, Anti-Inflamm. \& Anti-Aller. Agents in Med. Chem. 13 (1) (2014) 29-35.

[75] A. Astani, P. Schnitzler, Antiviral activity of monoterpenes beta-pinene and limonene against herpes simplex virus in vitro, Iranian J. Microbiol. 6 (3) (2014) 149-155.

[76] A. Hodi, I. Foldesi, J. Hajagos-Toth, E. Ducza, R. Gaspar, The effect of R(+) limonene on $\beta$-adrenerg signaling: the significance of oxidative stress index, Acta Pharmaceutica Hungarica 84 (3) (2014) 111-119.

[77] J. Hajagos-Toth, A. Hodi, A.B. Seres, R. Gaspar, Effects of d- and 1-limonene on the pregnant rat myometrium in vitro, Croatian Med. J. 56 (5) (2015) 431-438.

[78] J.A. Miller, J.E. Lang, M. Ley, R. Nagle, C.-H. Hsu, P.A. Thompson, C. Cordova, A. Waer, H.-H.S. Chow, Human breast tissue disposition and bioactivity of limonene in women with early-stage breast cancer, Cancer Prev. Res. 6 (2013) 577-584.

[79] K.M. Alle, S.M. Henshall, A.S. Field, R.L. Sutherland, Cyclin D1 protein is overexpressed in hyperplasia and intraductal carcinoma of the breast, Clin. Cancer Res. 4 (1998) 847-854.

[80] C.E. Gillett, A.H.S. Lee, R.R. Millis, D.M. Barnes, Cyclin D1 and associated proteins in mammary ductal carcinoma in situ and atypical ductal hyperplasia, J. Pathol. 184 (1998) 396-400.

[81] J.A. Miller, K. Pappan, P.A. Thompson, E.J. Want, A.P. Siskos, H.C. Keun, J. Wulff, C. Hu, J.E. Lang, H.-H.S. Chow, Plasma metabolomic profiles of breast cancer patients after short-term limonene intervention, Cancer Prev. Res. 8 (2015) 86-93.

[82] F. Labrie, V. Luu-The, C. Labrie, J. Simard, DHEA and its transformation into androgens and estrogens in peripheral target tissues: intracrinology, Front. Neuroendocrinol. 22 (2001) 185-212.

[83] K.E. Swales, M. Korbonits, R. Carpenter, D.T. Walsh, T.D. Warner, D. Bishop-Bailey, The farnesoid $\mathrm{X}$ receptor is expressed in breast cancer and regulates apoptosis and aromatase expression, Cancer Res. 66 (2006) 10120-10126. 